\title{
Avaliação do acesso ao tratamento de tuberculose sob a perspectiva de usuários
}

\author{
Evaluation of access to tuberculosis treatment from the \\ point of view of patients
}

\author{
Mara Cristina Ribeiro Furlan¹, Sonia Silva Marcon²
}

Resumo

Objetivo: Analisar, na perspectiva dos usuários, o acesso ao tratamento de tuberculose (TB) em serviços de saúde vinculados às Unidades Básicas de Saúde (UBS)/Unidades Saúde da Família (USF) e em ambulatórios de referência. Método: Estudo transversal, em que foram entrevistados 89 pacientes que realizavam o tratamento de TB no âmbito da $15^{\text {a }}$ Regional de Saúde do Paraná, com uso do questionário Primary Care Assessment Tool. Os dados foram submetidos à análise de variância. Resultados: As pessoas gastam menos com transporte quando realizam tratamento nas UBS/USF. No entanto, alguns indicadores, como marcação da consulta por telefone e espera média na consulta de retorno, são mais eficazes nos ambulatórios de referência. A visita domiciliar é realizada de forma insatisfatória em ambos os tipos de serviços. Conclusão: Há muitos desafios a serem enfrentados para que o acesso ao tratamento de TB consiga ser satisfatório tanto nas UBS/USF quanto nos serviços de referência.

Palavras-chave: tuberculose; família; avaliação de serviços de saúde; acesso aos serviços de saúde; enfermagem.

\section{Abstract}

Objective: to analyze from the users perspective, the access to treatment for tuberculosis (TB) in health services linked to Basic Health Units (UBS)/Family Health Units (FHU) and in referral outpatient clinics. Methods: Cross-sectional study, including interview with 89 patients who underwent tuberculosis treatment at the $15^{\text {th }}$ Health Unit of Paraná, using the questionnaire Primary Care Assessment Tool. Data were submitted to analysis of variance. Results: Show that people spend less money with transportation when they are treated at the UBS/USF. On the other hand, indicators such as: scheduling via phone and the average waiting time in the follow-up visit is more effective in outpatient clinics of reference. A home visit is unsatisfactory in both types of services. Conclusion: There are challenges to be faced so that access to tuberculosis treatment is satisfactory both for UBS/USF and for referral services.

Keywords: tuberculosis; family; health services evaluation; health services accessibility; nursing.

\footnotetext{
'Programa de Pós-graduação em Saúde e Denvolvimento na Região Centro-Oeste, Universidade Federal do Mato Grosso do Sul (UFMS) - Três Lagoas (MS), Brasil. ²Departamento de Enfermagem, Universidade Estadual de Maringá (UEM) - Maringá (PR), Brasil.

Trabalho realizado na $15^{\circ}$ Regional de Saúde do Paraná - Maringá (PR), Brasil.

Endereço para correspondência: Mara Cristina Ribeiro Furlan, Av. Cap. Olinto Mancini, 1662 - Colinos, CEP: 79600-080 - Três Lagoas (MS),

Brasil-Email: maracristina.mga@gmail.com

Fonte de financiamento: Fundação Araucária.

Conflito de interesses: nada a declarar.
} 


\section{INTRODUÇÃO}

A tuberculose (TB), apesar de ser uma doença passível de cura, ainda constitui um grave problema de saúde pública. No país, no período de 2005 a 2014, foram diagnosticados 73 mil novos casos de TB - só em 2013 ocorreram 4.577 óbitos $^{1}$. Devido às altas taxas de morbimortalidade causadas pela $\mathrm{TB}$, medidas para controlar a doença foram estabelecidas, sistematizadas e intensificadas pelo Programa Nacional de Controle da Tuberculose (PNCT). Entre as prioridades do PNCT estão: controle da doença a partir da busca de sintomáticos respiratórios; diagnóstico precoce e adequado; cura de pelo menos $85 \%$ das pessoas em tratamento; taxa de abandono menor que $5 \%{ }^{2}$.

Porém, há vários fatores relacionados à dificuldade para $\mathrm{o}$ sucesso do tratamento de TB, tais como: consumo abusivo de álcool, desemprego, baixa escolaridade, abandono em tratamento anterior, resistência das cepas bacterianas causadoras da TB às drogas antituberculose, efeitos colaterais das medicações (dores articulares, cefaleia, astenia e distúrbios gastrintestinais), tratamento prolongado ${ }^{2,3}$.

Devem-se considerar também questões relacionadas aos serviços de saúde como barreiras para adesão ao tratamento, por exemplo, desorganização do trabalho em equipe, demora no atendimento, desumanização, falta de vínculo entre usuários e profissionais de saúde, ausência de busca ativa para diagnosticar novos casos e para os que abandonam o tratamento 4 .

Uma das estratégias recomendadas para aumentar a adesão ao tratamento de TB é o Tratamento Diretamente Observado (TDO), no qual o profissional treinado passa a observar a tomada da medicação do paciente desde o início do tratamento até a sua cura ${ }^{2}$. O PNCT recomenda, portanto, que as equipes da Estratégia Saúde da Família (ESF) incorporem o TDO em suas atividades, além de se responsabilizarem pelo desenvolvimento de ações de diagnóstico, prevenção e tratamento da $\mathrm{TB}^{2}$.

Nesse sentido, o Ministério da Saúde recomenda a descentralização das ações de controle da TB para o âmbito das Unidades Básicas de Saúde (UBS) e das Unidades Saúde da Família (USF). Essa mudança no Programa de Controle de Tuberculose (PCT) vem requerendo a adoção de pesquisas e metodologias que permitam avaliar o alcance dessa estratégia e o impacto dessas inovações na organização dos serviços da Atenção Primária à Saúde (APS) ${ }^{5}$.

$\mathrm{O}$ acesso ao tratamento é um dos aspectos organizacionais e de desempenho dos serviços de saúde de TB que deve ser avaliado, pois influencia no diagnóstico e tratamento do paciente. $\mathrm{O}$ acesso é um conceito multidimensional e diz respeito à primeira etapa do percurso realizado pelo usuário quando parte em busca da satisfação de uma necessidade de saúde ${ }^{6}$. $\mathrm{O}$ acesso se divide em: geográfico, organizacional e social ${ }^{7}$.

O acesso geográfico está relacionado à distância média entre a população e os recursos, ao uso de transporte e ao tempo gasto para obtenção de assistência em saúde. O acesso referente à organização do serviço de saúde inclui características e recursos que facilitam ou impedem os esforços das pessoas em receber os cuidados de uma equipe ${ }^{7}$.

Entre os aspectos socioeconômicos vinculados ao paciente, os quais se inter-relacionam ao acesso, estão inclusas as condições de vida e moradia dos doentes, como tipo e local de habitação, situação de emprego, renda familiar e posse de automóvel ${ }^{8}$.

Dessa forma, o acesso ao tratamento ao paciente com TB inclui as seguintes características: horário de funcionamento, forma de marcação de consulta, horas de disponibilidade da unidade de saúde, intervalo de tempo entre marcar e consultar, disponibilidade para visitas domiciliares, tratamento de TB na unidade de saúde mais próxima, perda do dia de trabalho ou compromissos devido à consulta de $\mathrm{TB}$, dificuldade para se deslocar até a unidade de saúde e disponibilidade de medicamentos durante o tratamento?.

Nesse contexto, estudos recentes demonstram que melhorias no acesso ao tratamento de TB são necessários ${ }^{10-13}$, principalmente relacionados à realização de visita domiciliar e à distribuição do vale-transporte aos pacientes.

Além disso, investigação realizada em Campina Grande, na Paraíba, demonstra que, apesar da utilização do TDO estar articulada à ESF, com possibilidade de desencadear mudanças na organização e no funcionamento dos serviços de saúde, as ações desenvolvidas pelos profissionais da saúde da família do município estudado ainda estão orientadas pela lógica do modelo clínico, centrada no procedimento e ato médico ${ }^{14}$. Em outras palavras, apenas a descentralização dos serviços de controle da TB não garantem melhor acesso do paciente ${ }^{14,15}$.

Diante do papel fundamental que o acesso tem no tratamento do indivíduo com TB e da necessidade de se avaliarem os serviços de saúde que passam por reorganização da rede assistencial, principalmente no tratamento de TB, em que há recente orientação para descentralização da assistência, o objetivo deste estudo foi analisar, na perspectiva dos usuários, $\mathrm{o}$ acesso ao tratamento para TB em serviços de saúde vinculados às UBS/USF e em ambulatórios de referência.

\section{MÉTODOS}

Estudo transversal, descritivo, realizado na $15^{\text {a }}$ Regional de Saúde (RS) do Paraná, que é conformada por 30 municípios e que tem como sede a cidade de Maringá. Os municípios da RS não são prioritários no controle da TB, porém 22 deles tinham pacientes com diagnóstico de TB no período de coleta dos dados. A RS possui sete municípios com atendimento centralizado em ambulatório de referência para o tratamento de TB. Nos demais municípios (23), o serviço de controle da TB foi descentralizado para as UBS/USF a partir de 2008 (Tabela 1). Porém, como a maioria dos municípios é de pequeno porte, 
Tabela 1. Distribuição de casos de tuberculose em cidades da $15^{a}$ Regional de Saúde do Paraná, entre janeiro e março de 2012 , e cobertura da Estratégia Saúde da Família, Paraná, 2012

\begin{tabular}{|c|c|c|c|c|c|c|}
\hline \multirow[t]{2}{*}{ Municípios } & \multicolumn{2}{|c|}{ População } & \multicolumn{2}{|c|}{ Casos de tuberculose } & \multirow[t]{2}{*}{$\begin{array}{c}\begin{array}{c}\text { Estratégia } \\
\text { Saúde da } \\
\text { Família (ESF) } \\
\text { implantada }\end{array} \\
\mathrm{N}\end{array}$} & \multirow[t]{2}{*}{$\begin{array}{c}\text { Proporção } \\
\text { de cobertura } \\
\text { populacional } \\
\text { estimada pela } \\
\text { ESF } \\
\%\end{array}$} \\
\hline & $\mathbf{N}$ & $\%$ & $\mathbf{N}$ & $\%$ & & \\
\hline \multicolumn{7}{|l|}{$\begin{array}{l}\text { Serviço de controle da } \\
\text { tuberculose centralizado }\end{array}$} \\
\hline Astorga & 24780 & 3,7 & 3 & 2,0 & 5 & 69,6 \\
\hline Colorado & 22452 & 3,4 & 5 & 2,0 & 6 & 92,2 \\
\hline Mandaguaçu & 20008 & 3,0 & 5 & 3,3 & 2 & 34,4 \\
\hline Mandaguari & 32855 & 4,9 & 5 & 2,0 & 6 & 63,2 \\
\hline Nova Esperança & 26684 & 4,0 & 5 & 3,3 & 6 & 77,5 \\
\hline Paiçandú & 36333 & 5,5 & 4 & 2,7 & 6 & 56,9 \\
\hline Sarandi & 83724 & 12,6 & 19 & 18,6 & 1 & 4,1 \\
\hline \multicolumn{7}{|l|}{$\begin{array}{l}\text { Serviço de controle da } \\
\text { tuberculose descentralizado }\end{array}$} \\
\hline Doutor Camargo & 5832 & 0,9 & 1 & 0,7 & 1 & 59,1 \\
\hline Floraí & 5032 & 0,8 & 1 & 0,7 & 1 & 68,5 \\
\hline Iguaraçu & 4012 & 0,6 & 1 & 0,7 & 1 & 85,9 \\
\hline Itaguajé & 4553 & 0,7 & 1 & 0,7 & 1 & 75,7 \\
\hline Itambé & 5981 & 0,9 & 5 & 3,3 & 2 & 100 \\
\hline Ivatuba & 3027 & 0,5 & 1 & 0,7 & 1 & 100 \\
\hline Marialva & 32209 & 4,8 & 3 & 2,0 & 6 & 64,2 \\
\hline Maringá & 362329 & 54,5 & 78 & 48,4 & 64 & 60,9 \\
\hline Ourizona & 3379 & 0,5 & 3 & 2,0 & 1 & 100 \\
\hline Paranacity & 10338 & 1,6 & 4 & 2,7 & - & - \\
\hline Presidente Castelo Branco & 4821 & 0,7 & 2 & 1,4 & 1 & 71,5 \\
\hline Santa Fé & 10552 & 1,6 & 1 & 0,7 & 1 & 32,7 \\
\hline Santo Inácio & 5276 & 0,8 & 1 & 0,7 & 1 & 65,3 \\
\hline São Jorge do Ivaí & 5521 & 0,8 & 1 & 0,7 & 1 & 62,5 \\
\hline Uniflor & 2475 & 0,4 & 2 & 1,4 & 1 & 100 \\
\hline
\end{tabular}

Fonte: Brasil ${ }^{16,17}$.

ainda existe serviço de referência mantido pelo Consórcio Público Intermunicipal de Saúde do Setentrião Paranaense (CISAMUSEP), com sede em Maringá, para casos de difícil diagnóstico e de TB multirresistente, nos quais os pacientes são encaminhados se constatada necessidade pela equipe de saúde. Contudo, o tratamento é realizado no município de residência dos pacientes, local em que foram entrevistados.

Os dados referentes aos pacientes em tratamento de TB foram obtidos no Sistema de Informação de Agravos de Notificações $(\text { SINAN })^{16}$, com a Coordenação de TB da $15^{\text {a }}$ RS do Paraná. Foram estabelecidos os seguintes critérios de inclusão para o estudo: pacientes maiores de 18 anos de idade; residentes em algum dos municípios da $15^{\mathrm{a}}$ RS do Paraná; realização de pelo menos um mês de tratamento de TB. Foram utilizados como critérios de exclusão: falta de capacidade cognitiva para responder ao questionário, doentes acompanhados no sistema prisional e abandono do tratamento.
No período de janeiro a março de 2012, foram notificados 151 casos de TB na $15^{\mathrm{a}} \mathrm{RS}$, dos quais 133 atenderam aos critérios de inclusão do estudo, enquanto 12 estavam realizando o tratamento em sistema prisional, três abandonaram o tratamento e três possuíam problema cognitivo.

O número de indivíduos incluídos no estudo foi determinado considerando prevalência de 50\%, nível de confiança de 90\% e margem de erro de $5 \%$, resultando em uma amostra de 89 pessoas, a qual foi definida aleatoriamente por técnica de amostragem estratificada proporcional segundo o tipo de serviço em que era realizado o tratamento de TB (UBS/UFS ou ambulatórios de referência). Para o sorteio dos pacientes a serem estudados, foi utilizada uma lista de números aleatórios gerada pelo programa Statistica 8.0 (StatSoft).

A coleta de dados foi realizada no período de abril a junho de 2012 no ambiente domiciliar dos participantes. Realizou-se contato telefônico, ocasião em que foram explicitados os objetivos do estudo e solicitada a participação do indivíduo. 
Em caso de concordância, foi agendada visita para realizar a entrevista. Após três tentativas de contato, os indivíduos foram procurados diretamente no domicílio. Nas duas situações, foi realizada uma breve explicação sobre os objetivos do estudo, a participação por meio de entrevista com resposta a um roteiro de perguntas e a opção de participar ou não, sem qualquer prejuízo para a assistência prestada nos serviços de saúde. As entrevistas foram realizadas por uma das pesquisadoras do estudo, previamente treinada, e todos os participantes assinaram o Termo de Consentimento Livre Esclarecido (TCLE).

Para coleta dos dados, foi utilizado o Primary Care Assessment Tool (PCAT) ${ }^{18}$. Esse instrumento, que avalia cada uma das dimensões da APS, foi adaptado e validado para o Brasil ${ }^{19} \mathrm{e}$, posteriormente, para a atenção à $\mathrm{TB}^{20}$. $\mathrm{O}$ instrumento inclui também perguntas sobre o perfil socioeconômico do doente, informações clínico-epidemiológicas e estado atual de saúde.

Neste estudo, foram utilizados os resultados de 11 variáveis sobre o acesso ao tratamento e duas sobre o elenco de serviços. As variáveis relacionadas ao acesso ao tratamento foram: consulta médica em menos de 24 horas, informações por telefone na unidade de saúde, marcação da consulta por telefone, recebimento de visita domiciliar durante o tratamento, realização do tratamento de TB na unidade de saúde mais próxima de casa, perda do dia de trabalho ou compromissos devido à consulta de TB, dificuldade para se deslocar até a unidade de saúde, utilização de transporte motorizado no deslocamento até a unidade de saúde, despesa com transporte até a unidade de saúde, falta de medicamentos durante o tratamento e espera média por consulta de retorno maior que 60 minutos. Já as variáveis que dizem respeito ao elenco de serviços estão relacionadas ao oferecimento de cesta básica e de vale-transporte.

Cada questão do instrumento que deu origem as variáveis permitia ao sujeito assinalar a frequência em que a situação específica ocorria (se aplicada ou não à sua situação individual). As respostas eram apresentadas em uma escala do tipo Likert, à qual foi atribuído um valor entre 0 e 5 . $O$ valor 0 representava a resposta não sei ou não se aplica, e os valores de 1 a 5 registravam o grau de preferência ou concordância com as afirmações: nunca, quase nunca, às vezes, quase sempre e sempre.

Os dados foram digitalizados no programa Excel 2003 e verificados possíveis equívocos, como erros na entrada ou omissão de respostas. Após isso, realizou-se a análise descritiva dos dados. Para tanto, inicialmente foi determinado um escore médio de cada questão, o qual correspondia à somatória das respostas de todos os pacientes entrevistados, dividido pelo total de respondentes. Esse escore foi classificado como insatisfatório (valores menores que três), regular (valores maiores que três e menores que quatro) e satisfatório (valores maiores ou iguais a quatro $)^{13,15,21}$.

Para comparar o acesso ao tratamento nas UBS/USF e ambulatórios de referência, os dados foram submetidos à análise de variância, com o uso do Teste F. A análise de variância foi aplicada às questões que satisfizeram as pressuposições de independência, homocedasticidade e normalidade. A homocedasticidade foi verificada pelo Teste de Levene. Quanto às análises que violaram os critérios para o uso da Anova, foi usada a análise de variância não paramétrica, ou seja, o Teste de Kruskall-Wallis. O nível de significância estatística adotado em todos os testes foi de $5 \%$.

O estudo foi desenvolvido em consonância com as diretrizes disciplinadas da Resolução n 196/96 do Conselho Nacional de Saúde, vigente à época da realização da investigação, e aprovado pelo Comitê Permanente de Ética em Pesquisa com Seres Humanos da Secretaria de Saúde do Estado do Paraná (Parecer no 423/2011).

\section{RESULTADOS}

Os participantes do estudo possuíam, em sua maioria, TB da forma pulmonar (82\%), estavam entre o terceiro e sexto mês de tratamento (51\%), eram do sexo masculino $(50,6 \%)$ e casados (56,2\%), tinham entre 30 a 49 anos $(51,7 \%)$, apresentavam renda familiar entre um e três salários mínimos $(52,8 \%)$,possuíam ensino fundamental incompleto $(59,6 \%)$ e moravam com uma a três pessoas $(58,4 \%)$. O percentual de pessoas que trabalhavam antes de possuir TB era de 78,7\%, com declínio para 41,6\% após a infecção (Tabela 2).

Quanto às variáveis relacionadas ao elenco de serviços, os resultados evidenciaram que as variáveis com menores médias foram o oferecimento de cesta básica e de vale-transporte, ou seja, avaliadas como insatisfatória em ambos os tipos de serviços, com diferença estatística não significativa, enquanto a variável visita domiciliar durante o tratamento, relacionada ao acesso ao tratamento, obteve a segunda menor média em ambos os tipos de serviços, porém com pior desempenho dos ambulatórios de referência, resultado estatisticamente significativo (Tabela 3).

No que se refere à obtenção de informações por telefone nas unidades de saúde, houve comportamento semelhante em ambos os tipos de serviços, apresentando índices insatisfatórios. No entanto, os itens realização do tratamento de TB na unidade de saúde mais próxima de casa, perda do dia de trabalho ou compromissos devido à consulta de TB e despesa com transporte até a unidade de saúde foram avaliados como regulares, com exceção do último caso, que foi avaliado como irregular para os pacientes que realizavam o tratamento nas UBS/USF e como insatisfatório para aqueles que realizavam o tratamento nos serviços centralizados de TB - neste caso, com diferença estatisticamente significativa.

A utilização de transporte motorizado no deslocamento até a unidade de saúde apresentou diferença estatisticamente significativa, sendo avaliado como satisfatório nos serviços das UBS/USF e insatisfatório nos ambulatórios de referência. Contudo, o indicador dificuldade para se deslocar até a unidade 
de saúde foi considerado satisfatório em ambos os tipos de serviços, sem diferença estatisticamente significante.

Quanto aos indicadores consulta médica em menos de 24 horas, marcação da consulta por telefone e espera média por consulta de retorno maior que 60 minutos, eles foram avaliados como satisfatórios nos serviços de referência e como regulares nas
UBS/USF, com os dois últimos índices apresentando diferenças estatisticamente significativas.

Por último, a falta de medicamentos durante o tratamento não foi algo que ocorreu para a maioria das pessoas com TB, tendo média semelhante e satisfatória nos dois tipos de serviços, ou seja, não houve diferença estatisticamente significativa.

Tabela 2. Características clínicas e sociodemográficas dos pacientes acometidos por tuberculose da $15^{a}$ Regional de Saúde do Paraná, 2012

\begin{tabular}{|c|c|c|c|c|c|c|}
\hline \multirow[t]{2}{*}{ Variáveis } & \multicolumn{2}{|c|}{$\begin{array}{c}\text { Pacientes em tratamento } \\
\text { nas Unidades Básicas de } \\
\text { Saúde/Unidades Saúde da } \\
\text { Família } \\
(\mathrm{N}=64)\end{array}$} & \multicolumn{2}{|c|}{$\begin{array}{c}\text { Pacientes em tratamento } \\
\text { nos ambulatórios de } \\
\text { referência } \\
(\mathrm{N}=25)\end{array}$} & \multicolumn{2}{|c|}{ Total } \\
\hline & $\mathbf{N}$ & $\%$ & $\mathbf{N}$ & $\%$ & $\mathbf{N}$ & $\%$ \\
\hline \multicolumn{7}{|l|}{ Forma clínica da tuberculose } \\
\hline Pulmonar & 50 & 78,1 & 23 & 92,0 & 73 & 82,0 \\
\hline Extrapulmonar & 14 & 21,9 & 2 & 8,0 & 16 & 18,0 \\
\hline \multicolumn{7}{|c|}{ Tempo de tratamento medicamentoso } \\
\hline$>1$ mês até 3 meses & 14 & 21,9 & - & - & 14 & 15,7 \\
\hline$>3$ meses até 6 meses & 31 & 48,4 & 15 & 60,0 & 46 & 51,7 \\
\hline$>6$ até 9 meses & 4 & 6,3 & 3 & 12,0 & 7 & 7,9 \\
\hline Concluído & 15 & 23,4 & 7 & 28,0 & 22 & 24,7 \\
\hline \multicolumn{7}{|l|}{ Sexo } \\
\hline Masculino & 30 & 46,9 & 15 & 60,0 & 45 & 50,6 \\
\hline Feminino & 34 & 53,1 & 10 & 40,0 & 44 & 49,4 \\
\hline \multicolumn{7}{|l|}{ Idade } \\
\hline $18-29$ anos & 8 & 12,5 & 4 & 16,0 & 12 & 13,5 \\
\hline 30-49 anos & 35 & 54,7 & 11 & 44,0 & 46 & 51,7 \\
\hline 50-59 anos & 14 & 21,9 & 9 & 36,0 & 23 & 25,8 \\
\hline 60 anos ou mais & 7 & 10,9 & 1 & 4,0 & 8 & 9,0 \\
\hline \multicolumn{7}{|l|}{ Estado civil } \\
\hline Solteiro & 18 & 28,1 & 6 & 24,0 & 24 & 27,0 \\
\hline Casado & 33 & 51,6 & 17 & 68,0 & 50 & 56,2 \\
\hline Separado/divorciado & 9 & 14,1 & 1 & 4,0 & 10 & 11,2 \\
\hline Viúvo & 4 & 6,3 & 1 & 4,0 & 5 & 5,6 \\
\hline \multicolumn{7}{|l|}{ Trabalhava antes da tuberculose } \\
\hline Sim & 48 & 75,0 & 22 & 88,0 & 70 & 78,7 \\
\hline Não & 16 & 25,0 & 3 & 12,0 & 19 & 21,3 \\
\hline \multicolumn{7}{|c|}{ Continuou trabalhando depois da tuberculose } \\
\hline Sim & 26 & 40,6 & 11 & 44,0 & 37 & 41,6 \\
\hline Não & 38 & 59,4 & 14 & 56,0 & 52 & 58,4 \\
\hline \multicolumn{7}{|l|}{ Renda familiar } \\
\hline Sem renda & 3 & 4,7 & 1 & 4,0 & 4 & 4,5 \\
\hline Até 1 salário mínimo (SM) & 15 & 23,4 & 4 & 16,0 & 19 & 21,3 \\
\hline$>1$ até $3 \mathrm{SM}$ & 33 & 51,6 & 14 & 56,0 & 47 & 52,8 \\
\hline$>3$ até $5 \mathrm{SM}$ & 5 & 7,8 & 4 & 16,0 & 9 & 10,1 \\
\hline$>5 \mathrm{SM}$ & 8 & 12,5 & 2 & 8,0 & 10 & 11,2 \\
\hline \multicolumn{7}{|l|}{ Escolaridade } \\
\hline Sem escolaridade & 1 & 1,6 & 1 & 4,0 & 2 & 2,2 \\
\hline Ensino fundamental incompleto & 40 & 62,5 & 17 & 68,0 & 53 & 59,6 \\
\hline Ensino fundamental completo & 4 & 6,3 & - & - & 4 & 4,5 \\
\hline Ensino médio incompleto & 7 & 10,9 & 3 & 12,0 & 10 & 11,2 \\
\hline Ensino médio completo & 7 & 10,9 & 3 & 12,0 & 10 & 11,2 \\
\hline Ensino superior incompleto & 1 & 1,6 & 1 & 4,0 & 2 & 2,2 \\
\hline Ensino superior completo & 4 & 6,3 & - & - & 4 & 4,5 \\
\hline \multicolumn{7}{|c|}{ Número de pessoas que moram na casa } \\
\hline 1 a 3 & 34 & 53,1 & 18 & 72,0 & 52 & 58,4 \\
\hline 4 ou mais & 30 & 46,9 & 7 & 28,0 & 37 & 41,6 \\
\hline
\end{tabular}


Tabela 3. Análise de variância das variáveis de acesso ao tratamento e elenco de serviços de tuberculose nas Unidades Básicas de Saúde/ Unidades Saúde da Família e em ambulatórios de referência da 15ª Regional de Saúde do Paraná, 2012

\begin{tabular}{|c|c|c|c|c|c|}
\hline \multirow[t]{2}{*}{ Variáveis } & \multicolumn{2}{|c|}{$\begin{array}{l}\text { Paciente em tratamento na } \\
\text { Unidade Básica de Saúde } \\
\text { Unidades Básicas de Saúde/ } \\
\text { Unidades Saúde da Família } \\
\text { (n=64) }\end{array}$} & \multicolumn{2}{|c|}{$\begin{array}{l}\text { Paciente em tratamento no } \\
\text { ambulatório de referência } \\
\qquad(\mathrm{n}=25)\end{array}$} & \multirow[t]{2}{*}{$P$-valor } \\
\hline & Média & $\mathrm{DP}^{\mathbf{a}}$ & Média & $\mathrm{DP}^{\mathbf{a}}$ & \\
\hline Consulta médica em menos de 24 horas $^{\mathrm{b}}$ & 3,50 & 0,23 & 4,00 & 0,35 & 0,2577 \\
\hline Informações por telefone na unidade de saúde ${ }^{b}$ & 2,44 & 0,39 & 2,00 & 0,98 & 0,6807 \\
\hline Marcação de consulta por telefone $\mathrm{e}^{\mathrm{b}}$ & 3,62 & 0,78 & 4,37 & 0,74 & $0,0001^{\mathrm{d}}$ \\
\hline Recebimento de visita domiciliar durante o tratamento ${ }^{\mathrm{b}}$ & 2,37 & 0,18 & 1,36 & 0,29 & $0,0010^{\mathrm{d}}$ \\
\hline $\begin{array}{l}\text { Realização do tratamento de tuberculose na unidade de } \\
\text { saúde mais próxima de casa }{ }^{\text {b }}\end{array}$ & 3,93 & 0,20 & 2,20 & 0,32 & $<0,001^{\mathrm{d}}$ \\
\hline $\begin{array}{l}\text { Perda do dia de trabalho ou compromissos devido à } \\
\text { consulta de tuberculose }\end{array}$ & 3,68 & 0,22 & 2,52 & 0,36 & $0,0074^{\mathrm{d}}$ \\
\hline Dificuldade para se deslocar até a unidade de saúde ${ }^{c}$ & 4,59 & 0,22 & 4,12 & 0,36 & 0,1926 \\
\hline $\begin{array}{l}\text { Utilização de transporte motorizado no deslocamento até } \\
\text { a unidade de saúde }\end{array}$ & 3,34 & 0,23 & 2,12 & 0,37 & $0,0077^{\mathrm{d}}$ \\
\hline Despesa com transporte até a unidade de saúde ${ }^{c}$ & 4,04 & 0,22 & 2,92 & 0,35 & $0,0081^{\mathrm{d}}$ \\
\hline Falta de medicamentos durante o tratamento ${ }^{c}$ & 4,57 & 0,11 & 4,68 & 0,18 & 0,6385 \\
\hline Espera média por consulta de retorno maior que 60 minutos $^{c}$ & 3,58 & 0,18 & 4,76 & 0,29 & $0,0021^{\mathrm{d}}$ \\
\hline Oferecimento de cesta básica ${ }^{\mathrm{b}}$ & 1,12 & 0,07 & 1,24 & 0,12 & 0,4345 \\
\hline Oferecimento de vale-transporte $^{\mathrm{b}}$ & 1,12 & 0,06 & 1,08 & 0,11 & 0,7320 \\
\hline
\end{tabular}

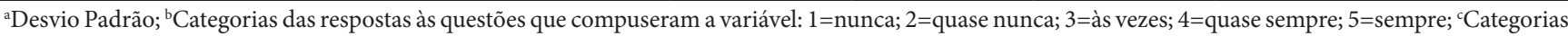

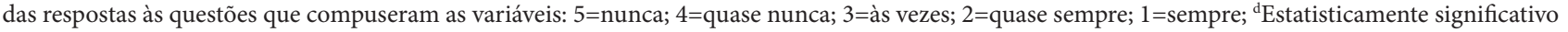

\section{DISCUSSÃO}

Os resultados encontrados demonstram, assim como em outros estudos ${ }^{3,22}$, que a TB ainda acomete, na maior parte dos casos, pessoas em vulnerabilidade social, ou seja, aquelas com condições socioeconômicas precárias, com baixa escolaridade, alta densidade domiciliar e renda baixa. Neste estudo, os doentes que vivem com uma a duas pessoas são maioria, porém o percentual daquelas que convivem com três a quatro pessoas é de 41,6\%.

Quanto à idade, os adultos jovens são os mais atingidos pela doença, o que indica a ocorrência de transmissão recente ${ }^{3}$. Nos países em desenvolvimento, $80 \%$ das pessoas com TB possuem entre 15 e 59 anos; estão, portanto, na faixa de produtividade social. A TB atinge, sobretudo, os homens em idade produtiva. Neste estudo, o percentual de pessoas que trabalhavam antes de possuir TB era de 78,7\%, com declínio para 41,6\% após o diagnóstico. A infecção pela doença faz com que muitos não tenham condições de trabalhar, e isso agrava a situação econômica, acarretando transtornos e dificuldade para toda a família ${ }^{22}$. Além do mais, o desemprego constitui preditor importante de abandono do tratamento ${ }^{15}$. Esses aspectos demonstram a importância do oferecimento de subsídios para que os pacientes possam aderir ao tratamento.

No entanto, em ambos os serviços, o oferecimento de cesta básica e de vale-transporte foram os indicadores avaliados com as piores médias, com ausência de diferença estatisticamente significativa. Estudo realizado em cinco municípios prioritários para o controle da TB das regiões Sudeste e Nordeste também demonstrou que as piores médias em relação ao acesso ao tratamento de TB estão relacionadas ao oferecimento de vale-transporte pelos serviços de saúde. Porém, o oferecimento de cestas básicas foi avaliado como satisfatório nesses mesmos serviços ${ }^{23}$.

Quando se reconhece a influência de fatores socioeconômicos na adesão ao tratamento de TB, percebe-se que, mesmo quando o tratamento é gratuito, a ausência de benefícios, ou de suporte, para cobrir gastos com transporte e comida pode dificultar a continuidade do tratamento ${ }^{23}$. Contudo, o oferecimento desse tipo de suporte exige dos serviços de saúde maior articulação com outros equipamentos de assistência social e exemplifica a inter-relação da acessibilidade organizacional e econômica ${ }^{3}$.

Outro fator preocupante é a ausência de visita ao doente de TB. Os serviços das UBS/ESF alcançaram maiores médias na avaliação desse quesito do que os ambulatórios de referência, porém, nos dois tipos de serviços, a avaliação a esse aspecto se mostrou insatisfatória, assim como em estudo realizado em Campina Grande, na Paraíba ${ }^{10}$. Contudo, pesquisa realizada em Ribeirão Preto, interior de São Paulo, utilizando o mesmo instrumento para avaliação, demonstrou desempenho regular ou satisfatório desse indicador em UBS/ESF e ambulatórios de referência ${ }^{13}$. Cabe destacar que a visita domiciliar é ferramenta inerente às equipes da ESF, portanto o indicador de acesso deveria ser maior nesse tipo de serviço. 
Estudo realizado em Ribeirão Preto, em quatro serviços de saúde que realizam o PCT, destacou que, para a realização de visitas domiciliares, é preciso reorganização das atividades internas dos serviços de saúde, pois elas são realizadas por profissionais que relatam falta de tempo hábil para um bom atendimento, já que, além das atividades externas, têm que garantir continuidade das atividades executadas no âmbito interno dos serviços ${ }^{24}$. Além disso, constatou que, para realização da visita domiciliar, é necessária a existência de estrutura mínima, como carro, motorista e profissional de saúde supervisor, o que alguns serviços de saúde podem não dispor em quantidade que permita atender às suas necessidades ${ }^{24}$.

Para os serviços de saúde centralizados de TB, há maior dificuldade para realização de visitas domiciliares para supervisão do tratamento por possuírem elevado número de doentes por TB e área de maior abrangência ${ }^{23}$.

A visita domiciliar, no entanto, é fundamental no combate à $\mathrm{TB}$, pois, além do objetivo de supervisionar a ingesta da medicação, visa a outros aspectos, como avaliação dos contatos, apoio psicossocial, fortalecimento de vínculo e maior orientação. Esses elementos são fundamentais para a adesão ao tratamento e prevenção da doença no contexto familiar ${ }^{21,23}$.

Por causa das dificuldades mencionadas, os ambulatórios de referência podem utilizar outras estratégias para melhorar o vínculo do paciente com os serviços de saúde e realização do TDO, como a supervisão da tomada da medicação na própria unidade de saúde ou de forma compartilhada, ou seja, o paciente recebe a consulta médica no ambulatório e realiza o TDO em outra unidade de saúde, mais próxima de seu domicílio ou trabalho ${ }^{2}$. Além disso, excepcionalmente, quando não for possível realizar nenhuma dessas alternativas, para garantir o TDO, a unidade pode propor ao doente que a observação seja realizada por uma pessoa da família ou da comunidade, previamente treinada, ou supervisionada por profissional de saúde ${ }^{2}$.

Em relação aos indicadores de acesso geográfico e econômico, como realização do tratamento de TB na unidade de saúde mais próxima de casa, utilização de transporte motorizado no deslocamento até a unidade de saúde, despesa com transporte até a unidade de saúde e perda de dia de trabalho ou compromisso, eles tiveram avaliações melhores nas UBS/USF. A avaliação do item dificuldade para se deslocar até a unidade de saúde foi satisfatória nos dois serviços, tendo em vista que a maior parte dos doentes foi entrevistada entre o terceiro e sexto mês de tratamento, quando vários sintomas da doença já haviam desaparecido.

O fator geográfico e socioeconômico é parte importante da estrutura conceitual da utilização de serviços de saúde pela população, já que pode impedir o doente de buscar o atendimento quando não possui recursos para pagar transporte e está com dificuldades para se locomover ${ }^{8,25}$.
A perda do dia de trabalho ou de compromissos devido à consulta de TB, que teve melhor avaliação nas UBS/USF, pode estar relacionada à maior flexibilidade dos serviços de atenção primária, já que os ambulatórios de referência, geralmente, têm horários fixos para o atendimento.

No entanto, a demora no atendimento e os horários incompatíveis com os turnos de trabalho podem fazer com que muitos indivíduos deixem de procurar o serviço de saúde, facilitando o abandono do tratamento pelo receio de perder o emprego diante da necessidade de faltas e atrasos ${ }^{23}$.

Os indicadores relacionados à acessibilidade organizacional, como possibilidade de marcar consulta por telefone e espera média na consulta de retorno maior que 60 minutos, tiveram melhores avaliações por usuários que fizeram o tratamento nos ambulatórios de referências do que pelos que realizaram nas UBS/USF, corroborando resultados de outro estudo ${ }^{23}$, o que pode ser explicado pelo fato de esses serviços possuírem equipe própria, com dias e/ou horários específicos para a assistência às pessoas com TB. Nesses serviços, após o ingresso do paciente no PCT, todo atendimento é realizado diretamente por essa equipe, e isso facilita a obtenção de consultas e o fluxo do atendimento no cenário dos serviços de saúde.

Esses aspectos diminuem a incidência do abandono ao tratamento de TB, já que a equipe de saúde está acessível a orientar, avaliar e acompanhar o estado de saúde do portador de TB em tratamento. Essa interação com os profissionais do PCT é importante, pois os efeitos colaterais e dúvidas apresentados pelos pacientes podem ocasionar consultas extras ${ }^{25}$.

$\mathrm{O}$ indicador falta de medicamentos durante o tratamento foi avaliado como satisfatório e obteve maior média entre os demais, com ausência de diferença estatisticamente significativa entre os serviços. Esse fato também foi evidenciado como potencialidade dos serviços de saúde de controle à $\mathrm{TB}$ em estudo realizado na Paraíba ${ }^{5}$. Assim, pressupõe-se que os esquemas terapêuticos custeados pelo governo e distribuídos de forma gratuita constituem um dos fatores que favorece a adesão dos pacientes ao tratamento e também facilita o processo de trabalho dos profissionais de saúde.

De modo geral, as UBS/USF e os ambulatórios de referência possuem desafios a enfrentar em relação ao acesso aos serviços de TB. Assim como este estudo, uma investigação realizada em Maringá, com objetivo de avaliar os serviços de saúde de TB na perspectiva dos pacientes, porém com enfoque na família, demostrou que os profissionais que trabalham no controle da TB, tanto em ambulatórios de referência quanto nas UBS, ainda não alcançaram o desempenho esperado no desenvolvimento de ações de controle da TB e que a descentralização do tratamento para as UBS, por si só, não constituiu um fator significativo que resultou na melhoria da qualidade do atendimento prestado aos usuários ${ }^{15}$. 
A principal limitação do estudo diz respeito ao fato de avaliar os serviços de controle da TB de diversas cidades, pois cada uma está inserida em um tipo de contexto e possui singularidades que não puderam ser totalmente exploradas devido à extensão do trabalho. Contudo, o agrupamento dos municípios em UBS/USF e em ambulatórios de referência possibilitou visualizar características importantes desses tipos de serviços.

\section{CONCLUSÃO}

Os resultados deste estudo mostraram que, no âmbito da $15^{a}$ RS do Paraná, as UBS/ESF tiveram as melhores avaliações quanto aos seguintes indicadores de acesso aos serviços de TB, quando comparadas aos ambulatórios de referência: realização do tratamento de TB na unidade de saúde mais próxima de casa, perda do dia de trabalho ou compromissos devido à consulta de TB, utilização de transporte motorizado no deslocamento até a unidade de saúde e despesa com transporte até a unidade de saúde, porém somente o último foi avaliado como satisfatório pelos usuários, enquanto os demais, como regulares.

No entanto, os ambulatórios de referência foram mais bem avaliados em relação aos indicadores: marcação da consulta por telefone e espera média por consulta de retorno maior que 60 minutos, ambos avaliados como satisfatórios.

Em termos gerais, $\mathrm{o}$ acesso ao tratamento de TB tem muitos desafios para que consiga ser satisfatório em todos os aspectos, tanto nas UBS/USF quanto nos serviços de referência. Os piores indicadores avaliados em ambos os tipos de serviços foram: oferecimento de cesta básica e de vale-transporte e recebimento de visita domiciliar.

Assim, é necessário que cada aspecto de acesso ao serviço de saúde seja estudado e que sejam implementadas ações para que os pontos negativos de cada tipo de serviço possam ser eliminados. Apenas descentralizar o serviço não foi suficiente para que houvesse satisfação dos usuários em relação ao acesso ao tratamento de TB.

A implementação de visitas domiciliares é um desafio às equipes de saúde. Além do empenho destas, é necessário que a gestão forneça subsídios, como qualificação profissional, materiais e tempo para sua implantação, o que acarretará em melhora dos indicadores de acesso ao PCT.

Espera-se que mais estudos sobre o tema sejam realizados em ambos os tipos de serviços para orientar as práticas de controle da TB com benefícios ao paciente.

\section{REFERÊNCIAS}

1. Brasil. Ministério da Saúde. Boletim Epidemiológico n.09/2015. Brasília: Ministério da Saúde; 2015.

2. Brasil. Ministério da Saúde. Manual de recomendações para o controle de tuberculose no Brasil. Brasília: Ministério da Saúde; 2011.

3. Furlan MCR, Oliveira SP, Marcon SS. Fatores associados ao abandono do tratamento de tuberculose no estado do Paraná. Acta Paul Enferm. 2012; 25(N. esp1.):108-14

4. Hino P, Cunha TN, Villa TCS, Santos CB. Perfil dos casos novos de tuberculose notificados em Ribeirão Preto (SP) no período de 2000 a 2006 Ciênc Saúde Coletiva. 2011; 16(Suppl 1):1295-1301

5. Marcolino ABL, Nogueira JA, Ruffino-Netto A, Moraes RM, Sá LD, Villa TCS, et al. Avaliação do acesso às ações de controle da tuberculose no contexto das equipes de saúde da família de Bayeux - PB. Rev Bras Epidemiol. 2009;12(2):144-57. http://dx.doi.org/10.1590/S1415-790X2009000200005.

6. Starfield B. Atenção Primária: equilíbrio entre necessidades de saúde, serviços e tecnologia [Internet]. Brasília: UNESCO/ Ministério da Saúde; 2002 [acesso em 2017 mar 7]. Disponível em: http://www.dominiopublico. gov.br/pesquisa/DetalheObraForm.do?select_action=\&co_obra $=14609$

7. Donabedian A. Aspects of Medical Care Administration. Boston: Haward University Press, 1973.

8. Assis MMA, Jesus WLA. Acesso aos serviços de saúde: abordagens, conceitos, políticas e modelo de análise. Ciênc Saúde Coletiva. 2012;17(11):2865-75.

9. Brasil. Conselho Nacional de Secretários de Saúde. Atenção Primária e Promoção da Saúde. Brasília: CONASS; 2007
10. Figueiredo TMRM, Villa TCS, Scatena LM, Cardozo GRI, Ruffino-Netto A, Nogueira JÁ, et al. Desempenho da atenção básica no controle da tuberculose. Rev Saude Publica. 2009;43(5):825-31. PMid:19768237. http:// dx.doi.org/10.1590/S0034-89102009005000054.

11. Lemos EF, Alves AMS, Oliveira GC, Rodrigues MP, Martins NDG, Croda $\mathrm{J}$. Health-service performance of TB treatment for indigenous and nonindigenous populations in Brazil: a cross-sectional study. BMC Health Serv Res. 2014;14(1):237. PMid:24885134. http://dx.doi.org/10.1186/1472-696314-237.

12. Arakawa T, Arcêncio RA, Scatolin BE, Scatena LM, Ruffino-Netto A, Villa TCS. Acessibilidade ao tratamento de tuberculose: avaliação de desempenho de serviços de saúde. Rev. Latino-Am. Enfermagem. 2011;19(4):994-1002. http://dx.doi.org/10.1590/S0104-11692011000400019.

13. Arakawa T, Arcêncio RA, Scatolin BE, Scatena LM, Ruffino-Netto A, Villa TCS. Acessibilidade ao tratamento de tuberculose: avaliação de desempenho de serviços de saúde. Rev Latino-Am Enfermagem. 2011;19(4):1-9.

14. Clementino FS, Miranda FAN. Acessibilidade: identificando barreiras na descentralização do controle da tuberculose nas unidades de saúde da família. Rev Enferm UERJ. 2010;18:584-90.

15. Furlan MCR, Gonzales RIC, Marcon SS. Desempenho dos serviços de controle da tuberculose em municípios do Paraná: enfoque na família. Rev Gaúcha Enferm. 2015;36(N. esp.):102-10.

16. Brasil. Departamento de Informática do Sistema Único de Saúde. Sistema de Informação de Agravos e Notificações. Tuberculose Notificações registradas [Internet]. Brasília: DATASUS; 2012 [citado em 2012 set 14]. Disponível em: http://tabnet.datasus.gov.br/cgi/tabcgi.exe?sinannet/ cnv/tubercpr.def 
17. Brasil. Ministério da Saúde. Departamento de Atenção Básica. Equipes de Saúde, Paraná. Brasília: DATASUS; 2012 [acesso em 14 set 2012]. Disponível em: http://tabnet.datasus.gov.br/cgi/deftohtm.exe?cnes/cnv/equipepr.def

18. Macinko J, Starfield B, Shi L. The contribution of primary care systems to health outcomes within organization for economic cooperation and development (OECD) countries, 1970-1998. Health Serv Res. 2003;38(3):83165. PMid:12822915. http://dx.doi.org/10.1111/1475-6773.00149.

19. Macinko J, Almeida C. Validação de uma metodologia de avaliação rápida das características organizacionais e do desempenho dos serviços de atenção básica do Sistema Único de Saúde (SUS) em nível local. Brasília: Organização Pan-Americana da Saúde; 2006. (Série técnica desenvolvimento de sistemas e serviços de saúde, 10).

20. Villa TCS, Ruffino-Netto A. Questionário para avaliação de desempenho de serviços de atenção básica no controle da tuberculose no Brasil. J Bras Pneumol. 2009;35(6):610-2. PMid:19618041. http://dx.doi.org/10.1590/ S1806-37132009000600014.

21. Cecilio HPM, Higarashi IH, Marcon SS. Opinião dos profissionais de saúde sobre os serviços de controle da tuberculose. Acta Paul Enferm. 2015;28(1):19-25.
22. Chirinos NEC, Meirelles BHS. Fatores associados ao abandono do tratamento da tuberculose: uma revisão integrativa. Texto Contexto Enferm. 2011;20(3):399-406. http://dx.doi.org/10.1590/S010407072011000300023 .

23. Arakawa T, Arcêncio RA, Scatolin BE, Scatena LM, Ruffino-Netto A, Villa TCS. Acessibilidade ao tratamento de tuberculose: avaliação de desempenho de serviços de saúde. Rev Lat Am Enfermagem. 2011;19(4):994-1002. http:// dx.doi.org/10.1590/S0104-11692011000400019.

24. Gonzales RIC, Monroe AA, Assis EG, Palha PF, Villa TCS, RuffinoNetto A. Desempenho de serviços de saúde no tratamento diretamente observado no domicílio para controle da tuberculose. Rev Esc Enferm USP. 2008;42(4):628-34. PMid:19192895. http://dx.doi.org/10.1590/ S0080-62342008000400003.

25. Lafaiete RS, Silva CB, Oliveira MG, Motta MCS, Villa TCS. Investigação sobre o acesso ao tratamento de tuberculose em Itaboraí/RJ. Esc Anna Nery. 2011;15(1):47-53.

Recebido em: Maio 29, 2016 Aprovado em: Jul. 09, 2017 